International Journal of Pure and Applied Mathematics

Volume 93 No. 1 2014, 7-21

ISSN: 1311-8080 (printed version); ISSN: 1314-3395 (on-line version)

url: http://www.ijpam.eu

doi: http://dx.doi.org/10.12732/ijpam.v93i1.2

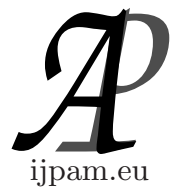

\title{
ON CLASS PRESERVING AUTOMORPHISMS OF SOME GROUPS OF ORDER $p^{6}$
}

\author{
Shiv Narain ${ }^{1}$, Ram Karan ${ }^{2}$ \\ ${ }^{1,2}$ Department of Mathematics \\ Kurukshetra University \\ Kurukshetra, 136 119, INDIA
}

\begin{abstract}
For an odd prime $p$ there are 43 isoclinism families of groups of order $p^{6}$ given by James [8]. Let $G$ be a group lying in these families.

We sort out those groups for which $\operatorname{Aut}_{c}(G)=\operatorname{Inn}(G)$. Let Autcent $(G)$ denotes the groups of all central automorphisms of $G$. We give an upper bound for $\left|A u t_{c}(G)\right|$ in terms of $\left|A u t_{c}(G) \cap \operatorname{Autcent}(G)\right|$ and $\mid A u t_{c}(G / Z(G) \mid$.
\end{abstract}

AMS Subject Classification: 20D45, 20D15

Key Words: finite p-groups, inner automorphism, class preserving automorphism, central automorphism, isoclinism

\section{Introduction}

We consider $G$ a finite p-group such that $|G|=p^{6}$ where p is an odd prime. Notations used are mostly standard, however for $x \in G, x^{G}$ denotes the conjugacy class of the element $x$ in $G$. An automorphism $f$ is called class preserving if $f(x) \in x^{G}$ for all $x \in G$. The set of all class preserving automorphisms is denoted by $A u t_{c}(G)$ and $\operatorname{Inn}(G)$ denotes the set of all inner automorphisms of $G$. Aut $t_{c}(G)$ forms a normal subgroup of $\operatorname{Aut}(G)$ and $\operatorname{Inn}(G)$ forms a normal subgroup of $A u t_{c}(G)$. The quotient group $A u t_{c}(G) / \operatorname{Inn}(G)$ is denoted by $\operatorname{Out}_{c}(G) . Z(G)$ denotes the center of $G$ and the Frattini subgroup

Received: August 20, 2013

(C) 2014 Academic Publications, Ltd.

$\S_{\text {Correspondence author }}$ url: www.acadpubl.eu 
of $G$ is denoted by $\Phi(G)$. An automorphisms $\phi$ of $G$ is said to be central if and only if it induces the identity automorphism on $G / Z(G)$, or equivalently, $g^{-1} \phi(g) \in Z(G)$ for all $g \in Z(G)$. The set of all central automorphisms of $\mathrm{G}$, denoted by Autcent $(G)$, forms a normal subgroup of $\operatorname{Aut}(\mathrm{G})$. It is well known that $\operatorname{Autcent}(G)=C_{\text {Aut }(G)}(\operatorname{Inn}(G))$.

Let $G$ be a group. A map $f: G \rightarrow G$ is called a co-cycle of $G$ if $f(x y)=$ $f(x) f(y)^{x}$, for all $x, y \in G$. Here $f(y)^{x}=x f(y) x^{-1}$. A co-cycle $f$ of $G$ is called a local co-boundary if for each $x \in G$ there exists an $a_{x} \in G$ such that $f(x)=a_{x}{ }^{-1} a_{x}{ }^{x}$. A co-cycle $f$ of $G$ is called a (global) co-boundary if there exists an $a \in G$ such that $f(x)=a^{-1} a^{x}$ for all $x \in G$. A group $G$ is said to enjoy Hasse's principle if every local co-boundary is a co-boundary. Ono, T [12] proved that a group $G$ enjoy's Hasses's principle if and only if every class preserving endomorphism is an inner automorphism. In the particular case when $G$ is a finite group, $G$ enjoys Hasse's principle if and only if $A u t_{c}(G)=\operatorname{Inn}(G)$.

Every abelian group enjoys the Hasse's principle trivially. Ono, T. and Wada, H [13] proved that the symmetric group $S_{n}$ and alternating group $A_{n}$ of degree $n$ enjoy Hasse's principle. They also proved that the dihedral group and the generalized quaternion group enjoys Hasse's principle. Further in this direction Kumar and Vermani [9] proved that a non-abelian finite p-group having a maximal subgroup which is cyclic enjoys Hasse's principle. They also proved that extra-special p-group enjoys Hasse's principle. In other notes (see [10], [11]) they showed that every p-group of order $p^{4}$ enjoys Hasse's principle. Fuma and Ninomiya [4] proved that a finite p-group having a cyclic subgroup of index $p^{2}$ enjoys Hasse's principle. Further using classification of groups of order $p^{5}$ (for an odd prime $p$ ) given by James [8], Yadav [15] showed that for an odd prime $p$ every -group of order $p^{5}$ except two isoclinism families enjoys Hasse's principle. We move a step ahead and using the classification given by James we try to sort out some of the groups of order $p^{6}$ (where $\mathrm{p}$ is an odd prime) for which $A u t_{c}(G)=\operatorname{Inn}(G)$. We find an upper bound for $\left|A u t_{c}(G)\right|$ in terms of $\left|\operatorname{Aut}_{c}(G) \cap \operatorname{Autcent}(G)\right|$ and $\left|A u t_{c}(G / Z(G))\right|$.

\section{Some Useful Results}

For a group $G$ and an abelian group $K$, Hom $(G, K)$ represents the group of all homomorphisms from $G$ to $K$. By $d(G)$ we mean the number of elements in a minimal generating set of $G$. A group $G$ is flat if every finite conjugacy class is a coset of a normal subgroup. The flatness condition is weaker than that of Camina pair (see [2], [3]). Let $G_{f}$ denotes the set of elements with finite 
conjugacy class in $G$. Tandra and Moran [14] showed that a group $G$ is flat if and only if $[x, G]$ is a subgroup of $G$ for all $x \in G_{f}$. A non abelian group $G$ is said to be purely non-abelian, if it has no non-trivial abelian direct factor. It is easy to see that a group is purely non abelian if its center is contained in the Frattini subgroup. The following lemma follows from [1].

Lemma 2.1. If $G$ is a purely non abelian finite group, then $\mid$ Autcent $(G) \mid=$ $\left|\operatorname{Hom}\left(G / \gamma_{2}(G), Z(G)\right)\right|$.

Lemma 2.2. [15] If $G$ is a finite p-group such that $Z(G) \subseteq[x, G]$, for all $x \in G-\gamma_{2}(G)$, then $\mid$ Aut $_{c}(G)|\geq|$ Autcent $(G)|| G / Z_{2}(G) \mid$.

The following lemma is proposition 14.4 of [7].

Lemma 2.3. Let $G$ be a finite group and $H$ be an abelian normal subgroup of $G$ such that $G / H$ is cyclic. Then $\operatorname{Out}_{c}(G)=1$ i.e. $\operatorname{Aut}_{c}(G)=\operatorname{Inn}(G)$.

Theorem 2.4. [15] Let $G$ be a finite p-group of class 2 such that $\gamma_{2}(G)$ is cyclic. Then $\operatorname{Out}_{c}(G)=1$.

Lemma 2.5. Let $A, B$ and $C$ be finite abelian groups. Then:

(i) $\operatorname{Hom}(A \times B, C) \cong \operatorname{Hom}(A, C) \times \operatorname{Hom}(B, C)$;

(ii) $\operatorname{Hom}(A, B \times C) \cong \operatorname{Hom}(A, B) \times \operatorname{Hom}(A, C)$

Lemma 2.6. Let $C_{m}, C_{n}$ be cyclic groups of order $m$ and $n$ respectively. Then $\operatorname{Hom}\left(C_{m}, C_{n}\right) \cong C_{d}$, where $d=$ g.c.d. $(m, n)$.

Lemma 2.7. [14] Let $G$ be a flat nilpotent group of order $p^{n}$ having nilpotency class $c$. Then $|Z(G)|>p$ if $n$ is even, and for any $k, 1 \leq k \leq c-1$, $\left|Z^{k+1}(G) / Z^{k}(G)\right|>p$.

For nilpotent groups of class $\geq 2$, we prove the following

Lemma 2.8. If $G$ is a nilpotent group of class $\geq 2$, then $\left|A u t_{c}(G)\right| \leq$ $\mid$ Aut $_{c}(G) \cap$ Autcent $(G)||$ Aut $_{c}(G / Z(G)) \mid$.

Proof. Observe that every class preserving automorphism $f$ in $\operatorname{Aut}_{c}(G)$ induces a class preserving automorphism $\bar{f}$ in $\operatorname{Aut}_{c}(G / Z(G))$. Then the map

$$
\alpha: \operatorname{Aut}_{c}(G) \rightarrow \operatorname{Aut}_{c}(G / Z(G))
$$

given by $\alpha(f)=\bar{f}$ is a homomorphism of groups with $\operatorname{Ker}(\alpha)=\operatorname{Aut}_{c}(G) \cap$ Autcent $(G)$. Hence $A_{\text {ut }}(G) / A_{u t}(G) \cap \operatorname{Autcent}(G) \cong$ a subgroup of $\operatorname{Aut}_{c}(G / Z(G))$. Now it follows that

$$
\left|A u t_{c}(G)\right| \leq\left|A u t_{c}(G) \cap \operatorname{Autcent}(G)\right| \cdot\left|A u t_{c}(G / Z(G))\right| .
$$


Corollary 2.9. If $G$ is a nilpotent group of class $\geq 2$, then $\left|A u t_{c}(G)\right| \leq$ $\mid$ Autcent $(G)|\cdot|$ Aut $_{c}(G / Z(G)) \mid$.

Note that if $A u t_{c}(G / Z(G))=\operatorname{Inn}(G / Z(G))$, then for each $\bar{f} \in A u t_{c}(G / Z(G))$, there exists an element $g Z \in G / Z(G)$ such that $\bar{f}(x Z)=g^{-1} x g Z$ for all $x Z \in G / Z$. Thus for each $\bar{f} \in A u t_{c}(G / Z(G))$, there exist some $T_{g} \in A u t_{c}(G)$ such that $\alpha\left(T_{g}\right)=\bar{f}$ and hence the homomorphism $\alpha$ is an epimorphism. But then $\operatorname{Aut}_{c}(G) / \operatorname{Aut}_{c}(G) \cap \operatorname{Autcent}(G) \cong \operatorname{Inn}(G / Z(G))$. Thus we have the following

Corollary 2.10. If $G$ is a nilpotent group of class $\geq 2$ such that

$$
\operatorname{Aut}_{c}(G / Z(G))=\operatorname{Inn}(G / Z(G)) \text {, }
$$

then

$$
\left|A u t_{c}(G)\right|=\left|A u t_{c}(G) \cap \operatorname{Autcent}(G)\right| \cdot\left|G / Z_{2}(G)\right| .
$$

Lemma 2.11. Let $G$ be a non-abelian finite p-group with $|Z(G)|=p$. Let $\left\{x_{1}, x_{2}, \ldots x_{r}\right\}$ be a minimal generating set for $G$. Suppose $Z(G)$ is not contained in $\left[x_{i}, G\right]$, for some $i, 1 \leq i \leq r$. Then Autcent $(G)$ is not contained in $A_{u t}(G)$.

Proof. Let $G$ be a non- abelian finite p-group with $|Z(G)|=p$. Then $G$ is purely non-abelian and hence $\mid$ Autcent $(G)|=| \operatorname{Hom}\left(G / \gamma_{2}(G), Z(G)\right) \mid$. Let $\left\{x_{1}, x_{2}, \ldots x_{r}\right\}$ be a minimal generating set for $G$. Then $G / \gamma_{2}(G)=<\overline{x_{1}}>\bigoplus<$ $\overline{x_{2}}>\bigoplus \cdots<\overline{x_{r}}>$ and hence

$$
\begin{aligned}
\mid \text { Autcent }(G) \mid & =\left|\operatorname{Hom}\left(\bigoplus_{i=1}^{r} \sum<\overline{x_{i}}>, Z(G)\right)\right| \\
& =p^{r}
\end{aligned}
$$

Define $H_{i}=Z(G) \cap\left[x_{i}, G\right]$. Clearly $H_{i}$ is a subgroup of $Z(G)$. Since $|Z(G)|=p$, it follows that either $H_{i}=\{e\}$ or $Z(G) \subseteq\left[x_{i}, G\right]$. Hence if $Z(G)$ is not contained in $\left[x_{i}, G\right]$, for some $i, 1 \leq i \leq r$, then $H_{i}=\{e\}$. Let if possible, Autcent $(G) \subseteq$ $A u t_{c}(G)$. Then every central automorphism is class preserving i.e. for each $\alpha \in \operatorname{Autcent}(G)$ and for every $x \in G$, we have $x^{-1} \alpha(x) \in Z(G) \cap[x, G]$. In particular

$$
\begin{aligned}
x_{i}^{-1} \alpha\left(x_{i}\right) & \in Z(G) \cap\left[x_{i}, G\right] \\
& =H_{i} \\
& =\{e\}
\end{aligned}
$$

Thus $\alpha\left(x_{i}\right)=x_{i}$ for every $\alpha \in \operatorname{Autcent}(G)$. But then

$$
\mid \text { Autcent }(G)|=| H o m\left(G / \gamma_{2}(G), Z(G)\right) \mid
$$




$$
\begin{aligned}
& =\left|\operatorname{Hom}\left(\bigoplus \sum_{j \boldsymbol{\sigma}_{i}}<\overline{x_{j}}>, Z(G)\right)\right| \\
& =p^{k} \quad \text { where } k<r
\end{aligned}
$$

This is a contradiction and hence the lemma holds.

Corollary 2.12. Let $G$ be a non-abelian finite p-group with $|Z(G)|=p$. Let $\left\{x_{1}, x_{2}, \ldots x_{r}\right\}$ be a minimal generating set for $G$ such that $Z(G)$ is not contained in $\left[x_{i}, G\right]$, for some $i, 1 \leq i \leq r$. Then

$$
\left|Z_{2}(G)\right| /|Z(G)| \leq\left|\operatorname{Aut}_{c}(G) \cap \operatorname{Autcent}(G)\right|<|\operatorname{Autcent}(G)|
$$

Proof. Let $G$ be a group as defined above, then by previous lemma $\mid A u t_{c}(G) \cap$ Autcent $(G)|<|$ Autcent $(G) \mid$. Since Autcent $(G)=C_{\text {Aut }(G)}(\operatorname{Inn}(G))$, therefore $Z(\operatorname{Inn}(G))=\operatorname{Inn}(G) \cap \operatorname{Autcent}(G) \subseteq \operatorname{Aut}_{c}(G) \cap \operatorname{Autcent}(G)$. Since $\operatorname{Inn}(G) \cong G / Z(G)$, therefore $|Z(G / Z(G))| \leq \mid$ Aut $t_{c}(G) \cap$ Autcent $(G) \mid$. Hence $\left|Z_{2}(G)\right| /|Z(G)| \leq \mid$ Aut $_{c}(G) \cap \operatorname{Autcent}(G)|<|$ Autcent $(G) \mid$.

\section{Isoclinic Groups}

The concept of isoclinic groups was introduced by P.Hall [6]. Let $X$ be a finite group and $\bar{X}=X / Z(X)$. Then commutation in $X$ gives a well-defined map $\alpha_{X}: \bar{X} \times \bar{X} \mapsto \gamma_{2}(X)$ such that $\alpha_{X}(x Z(X), y Z(X))=[x, y]$ for $(x, y) \in X \times X$. Two finite groups $G$ and $H$ are called isoclinic if there exist isomorphisms

$$
\begin{gathered}
\theta: G / Z(G) \rightarrow H / Z(H), \\
\phi: \gamma_{2}(G) \rightarrow \gamma_{2}(H),
\end{gathered}
$$

such that $\phi[\alpha, \beta]=\left[\alpha^{\prime}, \beta^{\prime}\right]$ for all $\alpha, \beta \in G$, where $\alpha^{\prime} Z(H)=\theta(\alpha(Z(G))$ and $\beta^{\prime} Z(H)=\theta(\beta Z(G))$. The resulting pair $(\theta, \phi)$ is called an isoclinism of $G$ onto $H$.

Before we start our work we state the following theorems proved by Yadav [15].

Theorem 3.1. Let $G$ and $H$ be two finite non-abelian isoclinic groups. Then $A_{u t}(G) \cong \operatorname{Aut}_{c}(H)$.

Theorem 3.2. Let $G$ be a finite p-group of order $p^{5}$, where $p$ is an odd prime. Then $\operatorname{Out}_{c}(G) \neq 1$ if and only if $G$ is isoclinic to the group in $\phi_{7}\left(1^{5}\right)$ and $\phi_{10}\left(1^{5}\right)$ the isoclinism family $\phi_{7}$ and $\phi_{10}$ and in these two cases $\left|A u t_{c}(G)\right|=p^{5}$. 
From the theorem 3.1, we conclude that it is sufficient to calculate $A u t_{c}(G)$ for only one member from each isoclinism family and from the theorem 3.2 , it is clear that if $G$ be any group of order $p^{5}$ ( $p$ is an odd prime), from the isoclinism families $\phi_{1}-\phi_{6}$ and $\phi_{8}-\phi_{9}$, then $\operatorname{Aut}_{c}(G)=\operatorname{Inn}(G)$.

Now we calculate $\left|A u t_{c}(G)\right|$ for some of the isoclinism families of groups of order $p^{6}$. The family $\phi_{1}$ corresponds to isoclinism family of abelian groups. For every abelian group we know that $A u t_{c}(G)=\operatorname{Inn}(G)$.

\section{Groups of Order $p^{6}$ Having a Cyclic Direct Factor}

In this section we consider those groups of order $p^{6}$ which are the direct product of a group of order $p^{5}$ and a cyclic group of order $p$. We consider the isoclinism families $\phi_{6}, \phi_{7}$ and $\phi_{10}$ in this section.

Theorem 4.1. Let $G$ be the group in the isoclinism familiy $\phi_{6}$. Then $\operatorname{Aut}_{c}(G)=\operatorname{Inn}(G)$.

Proof. Let $G$ be the group $\phi_{6}\left(1^{6}\right)$. Then $G=H \times(1)$, where $H=\phi_{6}\left(1^{5}\right)$. Since $H$ is a nilpotent group of class 3 with $|Z(H)|=p^{2}$, therefore $G$ is a nilpotent group of class 3 having $|Z(G)|=p^{3}$. Clearly $A_{u} t_{c}(G)=A_{u}(H)$. But $\left|A t_{c}(H)\right|=|\operatorname{Inn}(H)|=p^{3}(\operatorname{lemma} 5.3,[15])$ and hence $\left|A u t_{c}(G)\right|=|\operatorname{Inn}(G)|$.

Theorem 4.2. Let $G$ be the group in the isoclinism familiy $\phi_{7}$ and $\phi_{10}$. Then $\operatorname{Aut}_{c}(G) \neq \operatorname{Inn}(G)$.

Proof. Let $G$ be the group $\phi_{7}\left(1^{6}\right)$. Then $G$ is a direct product of $\phi_{7}\left(1^{5}\right)$ and and a cyclic group of order $p$. Similarly if $G$ is the group $\phi_{10}\left(1^{6}\right)$, then $G$ is a direct product of $\phi_{10}\left(1^{5}\right)$ and and a cyclic group of order $p$. In both of the cases $|Z(G)|=p^{2}$. Now from theorem 3.2, it follows that $\left|A u t_{c}(G)\right|=p^{5}$. Hence $\operatorname{Aut}_{c}(G) \neq \operatorname{Inn}(G)$, since $|\operatorname{Inn}(G)|=p^{4}$.

If $G$ is the group $\phi_{8}(42)$ in the isoclinism family $\phi_{8}$, then $G$ has an element of order $p^{4}$. Thus $\operatorname{Aut}_{c}(G)=\operatorname{Inn}(G)[4]$.

\section{Nilpotent Groups of Class 2 in which $\gamma_{2}(G)$ is Cyclic}

In this section we consider those groups of order $p^{6}$ which are nilpotent groups of class 2 , and $\gamma_{2}(G)$ is cyclic. We consider the isoclinism families $\phi_{2}, \phi_{5}$ and $\phi_{14}$ in this section.

Theorem 5.1. Let $G$ be the group in the isoclinism familiy $\phi_{2}$. Then $\operatorname{Aut}_{c}(G)=\operatorname{Inn}(G)$. 
Proof. Let $G$ be the group $\phi_{2}(411)_{b}$. Then $G$ has the following presentation $G=<\alpha, \alpha_{1}, \alpha_{2}, \gamma>$ such that $\left[\alpha, \alpha_{1}\right]=\alpha_{2}=\gamma^{p^{3}}, \alpha^{p}=\alpha_{1}^{p}=\alpha_{2}^{p}=1$.

Clearly $\gamma_{2}(G)=\left\langle\alpha_{2}\right\rangle$. Using the conventions we note that $\left[\alpha_{1}, \alpha_{2}\right]=$ $\left[\alpha, \alpha_{2}\right]=1$. Also $\left[\gamma, \alpha_{2}\right]=\left[\gamma, \gamma^{p^{3}}\right]=1$. This shows that $\alpha_{2} \in Z(G)$. But then $\gamma_{2}(G) \subseteq Z(G)$. Thus $G$ is a nilpotent group of class 2 such that $\gamma_{2}(G)$ is cyclic, therefore by theorem 2.4, Aut $t_{c}(G)=\operatorname{Inn}(G)$.

In the similar fashion we find that if $G$ is a group in the isoclinism families $\phi_{5}$ or $\phi_{14}$, then $\operatorname{Aut}_{c}(G)=\operatorname{Inn}(G)$.

\section{Groups of Order $p^{6}$ Having a Normal Abelian Subgroup}

In this section we calculate $A u t_{c}(G)$ for those groups $G$ of order $p^{6}$ when $G$ has a normal abelian subgroup $H$ such that $G / H$ is cyclic. We will study isoclinism families $\phi_{3}, \phi_{4}, \phi_{9}, \phi_{16}$ and $\phi_{35}$ in this section.

Theorem 6.1. Let $G$ be the group in the isoclinism families $\phi_{3}$. Then $\operatorname{Aut}_{c}(G)=\operatorname{Inn}(G)$.

Proof. Let $G$ be the group $\phi_{3}(3111)_{e}$. Then $G$ has the following presentation $G=<\alpha, \alpha_{1}, \alpha_{2}, \alpha_{3}>$ such that $\left[\alpha_{1}, \alpha\right]=\alpha_{2},\left[\alpha_{2}, \alpha\right]=\alpha_{3}, \alpha^{p}=\alpha_{1}^{p^{3}}=\alpha_{2}^{p}=$ $\alpha_{3}^{p}=1$. Here $\gamma_{2}(G)=<\alpha_{2}, \alpha_{3}>$. Let $H=<\alpha_{1}, \alpha_{2}, \alpha_{3}>$. Since $\gamma_{2}(G) \subseteq H$, therefore $H$ is a normal subgroup of $G$. Now $\left[\alpha_{1}, \alpha_{2}\right]=\left[\alpha_{1}, \alpha_{3}\right]=\left[\alpha_{2}, \alpha_{3}\right]=1$. This shows that $H$ is an abelian subgroup of $G$. Hence $H$ is a normal abelian subgroup of $G$ such that $G / H=\langle\alpha H>$ is a cyclic group of prime order. So by theorem 2.3, $\operatorname{Aut}_{c}(G)=\operatorname{Inn}(G)$.

On the same lines we find that if $G$ is a group from isoclinism families $\phi_{4}, \phi_{9}$, $\phi_{16}$ and $\phi_{35}$, then $\operatorname{Aut}_{c}(G)=\operatorname{Inn}(G)$.

\section{Groups of Order $p^{6}$ in which $\operatorname{Autcent}(G) \subseteq A u t_{c}(G)$}

We study the isoclinism families $\phi_{24}, \phi_{36}$ and $\phi_{38}$. For a group $G$ lying in these families, we prove that for these groups Autcent $(G) \subseteq A u t_{c}(G)$. To show this we use the fact that if $Z(G) \subseteq[x, G]$ for each $x \in G-\gamma_{2}(G)$, then $\operatorname{Autcent}(G) \subseteq \operatorname{Aut}_{c}(G)$. We find that for any group $G$ in these families $\operatorname{Aut}_{c}(G) \neq \operatorname{Inn}(G)$.

Theorem 7.1. Let $G$ be the group $\phi_{24}\left(1^{6}\right)$ in the isoclinism familiy $\phi_{24}$. Then $\left|A u t_{c}(G)\right|=p^{6}$ and therefore $\operatorname{Aut}_{c}(G) \neq \operatorname{Inn}(G)$. 
Proof. Let $G=\phi_{24}\left(1^{6}\right)$ be a group in the isoclinism family $\phi_{24}$. Then $G=<\alpha, \alpha_{1}, \alpha_{2}, \alpha_{3}, \alpha_{4}, \beta>$ where $\left[\alpha_{i}, \alpha\right]=\alpha_{i+1},\left[\alpha_{1}, \beta\right]=\alpha_{4}, \alpha^{p}=\alpha_{1}^{(p)}=\beta^{p}=$ $\alpha_{i+1}^{(p)}=1, \quad i=1,2,3$. Here $\gamma_{2}(G)=<\alpha_{2}, \alpha_{3}, \alpha_{4}>$ and $G$ is a nilpotent group of class 4 such that $|Z(G)|=p$. Using the presentation of the group we find that $Z(G)=<\alpha_{4}>$. Now it follows from $\S 4.1$ of [8], that $G / Z(G)=\phi_{3}\left(1^{5}\right)$. Gumber and Sharma ( theorem 4.2, [5]) showed that $\exp (G / Z(G))=p$ and $d(G)=3$. Since $Z(G) \subseteq \gamma_{2}(G)$, therefore $\exp \left(G / \gamma_{2}(G)\right)=p$. Now any element $x \in G$ can be written as $a \alpha^{l} \alpha_{1}^{m} \beta^{n}$, where $a \in \gamma_{2}(G)$ and $0 \leq l, m, n \leq p-1$. Note that $x \in G-\gamma_{2}(G)$ unless $\mathrm{l}=\mathrm{m}=\mathrm{n}=0$. We claim that for each $x \in G-\gamma_{2}(G)$, $Z(G) \subseteq[x, G]$.

Let $H=<\alpha_{1}, \alpha_{2}, \alpha_{3}, \alpha_{4}>$ and $K=<\alpha_{2}, \alpha_{3}, \alpha_{4}, \beta>$. Then $\gamma_{2}(G)$ is contained in $H$ and $K$. Thus $H$ and $K$ are normal abelian subgroups of $G$. Since $\gamma_{2}(G)$ and $H$ commutes elementwise, therefore for each $x \in G-\gamma_{2}(G)$, $[x, H]$ and $[x, K]$ are subgroups of $G$ contained in $[x, G]$.

Suppose $l \neq 0$, then using the facts that $\gamma_{2}(G)$ is abelian and it commutes with $H$ and $K$ elemenwise, we see that

$$
\left[x, \alpha_{3}\right]=\left[a \alpha^{l} \alpha_{1}^{m} \beta^{n}, \alpha_{3}\right]=\left[\alpha^{l}, \alpha_{3}\right]=\alpha_{4}^{-l}
$$

Since $p$ does not divides $l$, therefore $\alpha_{4}^{-l} \in[x, H]$ implies that $\alpha_{4} \in[x, H]$. Hence $Z(G) \subseteq[x, H]$.

Suppose $l=m=0$. Since $\gamma_{2}(G)$ and $H$ commute elementwise, therefore

$$
\left[x, \alpha_{1}\right]=\left[a \beta^{n}, \alpha_{1}\right]=\left[\beta^{n}, \alpha_{1}\right]=\alpha_{4}^{-n}
$$

Again using the same argument as above $Z(G) \subseteq[x, H]$.

If $l=n=0$. Since $\gamma_{2}(G)$ and $K$ commute elementwise, therefore

$$
[x, \beta]=\left[a \alpha_{1}^{m}, \beta\right]=\left[\alpha_{1}^{m}, \beta\right]=\alpha_{4}^{m}
$$

A similar argument shows that $Z(G) \subseteq[x, K]$.

Thus in each case discussed above either $Z(G) \subseteq[x, H] \subseteq[x, G]$ or $Z(G) \subseteq$ $[x, K] \subseteq[x, G]$ for each $x \in G-\gamma_{2}(G)$. But then Autcent $(G) \subseteq \operatorname{Aut}_{c}(G)$. We now calculate $\mid$ Autcent $(G) \mid$. Since $|Z(G)|=p$, therefore $G$ is purely nonabelian. Now it follows that

$$
\mid \text { Autcent }(G)|=| \operatorname{Hom}\left(G / \gamma_{2}(G), Z(G)\right) \mid=p^{3}
$$

Now $G / Z(G)=\phi_{3}\left(1^{5}\right)=\phi_{3}\left(1^{4}\right) \times(1)$ where

$$
\phi_{3}\left(1^{4}\right)=<\alpha, \alpha_{1}, \alpha_{2}, \alpha_{3} \mid\left[\alpha_{i}, \alpha\right]=\alpha_{i+1}, \alpha^{p}=\alpha_{i}^{(p)}=\alpha_{3}^{p}=1>i=1,2 .
$$


Let $L=\phi_{3}\left(1^{4}\right)$. Then $L$ is a non-abelian group of order $p^{4}$ with maximal class. Now it follows that $|Z(L)|=p$. But then $|Z(G / Z(G))|=p^{2}$ and hence $\left|Z_{2}(G)\right|=p^{3}$. By theorem 3.2, it follows that $A_{u t}(G / Z(G))=\operatorname{Inn}(G / Z(G))$. Hence by Corollary 2.10, we have

$$
\begin{aligned}
\mid \text { Aut }_{c}(G) \mid & =\mid \text { Aut }_{c}(G) \cap \operatorname{Autcent}(G)|| G / Z_{2}(G) \mid \\
& =\mid \text { Autcent }(G)|| G / Z_{2}(G) \mid \\
& =p^{6}
\end{aligned}
$$

Hence $\left|A u t_{c}(G)\right|=p^{6}$. Also $A u t_{c}(G) \neq \operatorname{Inn}(G)$ as $|\operatorname{Inn}(G)|=|G / Z(G)|=p^{5}$.

Let $G$ be the group $\phi_{36}\left(1^{6}\right)$ or $\phi_{38}\left(1^{6}\right)$ in the isoclinism families $\phi_{36}$ or $\phi_{38}$. Then both $G$ and $G / Z(G)$ are nilpotent groups of maximal class. In these cases $G / Z(G)$ is $\phi_{9}\left(1^{5}\right)$ and $\phi_{10}\left(1^{5}\right)$ respectively. Now using the theorem 3.2 and a similar argument as in the above theorem, we find that $A u t_{c}(G) \neq \operatorname{Inn}(G)$.

\section{Groups of Order $p^{6}$ in which $\operatorname{Autcent}(G)$ is Minimum i.e. $\operatorname{Autcent}(G)=Z(\operatorname{Inn}(G))$}

In this section we prove that if $G$ is a group from the isoclinism families $\phi_{25}$, $\phi_{26}, \phi_{28}, \phi_{29}$ and $\phi_{40}-\phi_{43}$, then $A_{u t}(G)=\operatorname{Inn}(G)$. To prove this we need the following theorem proved by Gumber and Sharma [5].

Theorem 8.1. If $G$ is a group of order $p^{6}$ with nilpotency class 3 or 4 , then Autcent $(G)=Z(\operatorname{Inn}(G))$ if and only if $G$ is isomorphic to one of the group in the isoclinism families $\phi_{25}, \phi_{26}, \phi_{28}, \phi_{29}$ and $\phi_{40}-\phi_{43}$.

Theorem 8.2. Let $G$ be the group in the isoclinism families $\phi_{25}, \phi_{26}, \phi_{28}$, $\phi_{29}$ and $\phi_{40}-\phi_{43}$. Then $\operatorname{Aut}_{c}(G)=\operatorname{Inn}(G)$.

Proof. Let $G$ be any group in the isoclinism families $\phi_{25}, \phi_{26}, \phi_{28}, \phi_{29}$ and $\phi_{40}-\phi_{43}$. Then form $\S 4.1$ of [8], it follows that $G / Z(G)$ is one of the following groups $\phi_{3}(221)_{b_{1}}, \phi_{3}(221)_{b_{\nu}}, \phi_{6}\left(1^{5}\right), \phi_{6}(221) b_{1 / 2(p-1)}$ and $\phi_{6}(221) d_{0}$. In either case we have $A_{u t}(G / Z(G))=\operatorname{Inn}(G / Z(G)$ ) (by theorem 3.2) and hence

$$
\begin{aligned}
\mid \text { Aut }_{c}(G) \mid & =\mid \text { Aut }_{c}(G) \cap \text { Autcent }(G)|\cdot| G / Z_{2}(G) \mid \\
& \leq \mid \text { Autcent }(G)|\cdot| G / Z_{2}(G) \mid
\end{aligned}
$$

But for these groups (by Theorem 8.1),

$$
\mid \text { Autcent }(G)|=| Z\left(\operatorname{Inn}(G)|=| Z_{2}(G)|/| Z(G) \mid .\right.
$$


Thus

$$
\left|A u t_{c}(G)\right| \leq|\operatorname{Autcent}(G)| \cdot\left|G / Z_{2}(G)\right|=\frac{\left|Z_{2}(G)\right|}{|Z(G)|} \cdot \frac{|G|}{\left|Z_{2}(G)\right|}=|\operatorname{Inn}(G)|
$$

Hence if $G$ is isomorphic to one of the groups in the isoclinism families $\phi_{25}, \phi_{26}$, $\phi_{28}, \phi_{29}$ and $\phi_{40}-\phi_{43}$. Then $\operatorname{Aut}_{c}(G)=\operatorname{Inn}(G)$.

\section{Nilpotent Groups of Class 3}

In this section we consider the isoclinism families $\phi_{31}-\phi_{34}$ and $\phi_{22}$. The group $G$ lying in any one of these families is a nilpotent group of class 3. Using the lemma 2.7 and the definition of flat groups we find that $A u t_{c}(G)=\operatorname{Inn}(G)$.

Theorem 9.1. Let $G$ be the group $\phi_{31}\left(1^{6}\right)$ in the isoclinism familiy $\phi_{31}$. Then $\operatorname{Aut}_{c}(G)=\operatorname{Inn}(G)$.

Proof. Let $G$ be the group $\phi_{31}\left(1^{6}\right)$. Then $G=<\alpha, \alpha_{1}, \alpha_{2}, \beta_{1}, \beta_{2}, \gamma>$ where $\left[\alpha_{i}, \alpha\right]=\beta_{i},\left[\alpha_{1}, \beta_{1}\right]=\gamma,\left[\alpha_{2}, \beta_{2}\right]=\gamma, \alpha^{p}=\alpha_{i}^{p}=\beta_{i}^{p}=\gamma^{p}=1, \quad i=1,2$. Note that $\gamma_{2}(G)=<\beta_{1}, \beta_{2}, \gamma>$ and $G$ is a nilpotent group of class 3 . It follows that $\gamma_{2}(G) \subseteq Z_{2}(G)$. Now $G / \gamma_{2}(G)$ is an elementary abelian group of order $p^{3}$. Now from $\S 4.1$ of [8], we find that $|Z(G)|=p$ and $G / Z(G)=$ $\phi_{4}\left(1^{5}\right)$. Hence by theorem 3.2 and Corollary 2.10, it follows that $\left|\operatorname{Aut}_{c}(G)\right|=$ $\mid$ Aut $_{c}(G) \cap \operatorname{Autcent}(G)|\cdot| G / Z_{2}(G) \mid$. Now we calculate $\mid A u t_{c}(G) \cap$ Autcent $(G) \mid$. Since $|Z(G)|=p$ and $|G|=p^{6}$, therefore by lemma $2.7 G$ can not be flat, But $G / Z(G)$ being a nilpotent group of class 2, is flat. This shows that there exists some $x_{1} \in G-Z(G)$ such that $Z(G)$ is not contained in $\left[x_{1}, G\right]$. Since $|Z(G)|=p$, therefore $x_{1} \in G-\gamma_{2}(G)$. Now $\left\{x_{1}\right\}$ can be extended to a minimal generating set say $\left\{x_{1}, x_{2}, x_{3}\right\}$ of $G$. But then by Corollary $2.12 \mid A u t_{c}(G) \cap$ Autcent $(G)|<|$ Autcent $(G) \mid$. i.e. $\left|A u t_{c}(G) \cap \operatorname{Autcent}(G)\right| \leq p^{2}$. Now $G$ has nilpotency class 3 , therefore $\gamma_{2}(G) \subseteq Z_{2}(G)$. Now

$$
\left|A u t_{c}(G)\right|=\left|\operatorname{Aut}_{c}(G) \cap \operatorname{Autcent}(G)\right| \cdot\left|G / Z_{2}(G)\right| \leq p^{2} \cdot p^{3}
$$

Thus $\left|A u t_{c}(G)\right| \leq p^{5}$. Since $\left|A u t_{c}(G)\right| \geq|\operatorname{Inn}(G)|=|G / Z(G)|=p^{5}$, therefore $\operatorname{Aut}_{c}(G)=\operatorname{Inn}(G)$.

If $G$ is a group in isoclnism families $\phi_{32}-\phi_{34}$ and $\phi_{22}$, then $G$ is a nilpotent group of class 3 with $|Z(G)|=p$. Using the presentations of these groups and the similar arguments as in the above theorem we find that $\operatorname{Aut}_{c}(G)=\operatorname{Inn}(G)$. 
10.

Theorem 10.1. Let $G$ be a nilpotent group of class $\geq 2$. Then $A u t_{c}(G) \cap$ Autcent $(G) \cong \operatorname{Hom}_{c}\left(G / \gamma_{2}(G), Z(G)\right)$.

Proof. Let $G$ be a nilpotent group of class $\geq 2$. Let $f \in A u t_{c}(G) \cap$ Autcent $(G)$. Then for each $x \in G, x^{-1} f(x) \in Z(G) \cap[x, G]$. Thus we can define a map $\alpha_{f}: G \rightarrow Z(G)$ by $\alpha_{f}(x)=x^{-1} f(x)$. Clearly $\alpha_{f}$ is a homomorphism of $G$ into $Z(G)$. Since $Z(G)$ is abelian, therefore $\alpha_{f}$ sends elements of $\gamma_{2}(G)$ to 1 . Therefore we have a homomorphism $\overline{\alpha_{f}}: G / \gamma_{2}(G) \rightarrow Z(G)$ given by $\overline{\alpha_{f}}\left(x \gamma_{2}(G)\right)=x^{-1} f(x)$. Now the map $f \mapsto \overline{\alpha_{f}}$ is a homomorphism of the group Aut $_{c}(G) \cap$ Autcent $(G)$ into $\operatorname{Hom}\left(G / \gamma_{2}(G), Z(G)\right)$. We denote this map by $\psi$. Now it is fairly easy to see that $\psi$ is a monomorphism of groups. We denote

$$
\left\{f \in \operatorname{Hom}\left(G / \gamma_{2}(G), Z(G)\right): f\left(x \gamma_{2}(G) \in Z(G) \cap[x, G] \forall x \in G\right\}\right.
$$

by $\operatorname{Hom}_{c}\left(G / \gamma_{2}(G), Z(G)\right)$. Now it is clear that if $f \in \operatorname{Aut}_{c}(G) \cap \operatorname{Autcent}(G)$, then $\overline{\alpha_{f}} \in H_{o m}\left(G / \gamma_{2}(G), Z(G)\right)$. On the other hand let

$$
f \in \mathrm{Hom}_{c}\left(G / \gamma_{2}(G), Z(G)\right)
$$

then we can define a map $\phi: G \rightarrow G$ by $\phi(x)=x f\left(x \gamma_{2}(G)\right)$. Since $f\left(x \gamma_{2}(G)\right) \in$ $Z(G) \cap[x, G]$, therefore for each $x \in G$ there exists some $a \in G$ such that $f\left(x \gamma_{2}(G)\right)=[x, a]$. But then $\phi(x)=x f\left(x \gamma_{2}(G)\right)=x[x, a]=a^{-1} x a$. Thus $\phi$ is a class preserving homomorphism. Since $G$ is finite, $\phi \in A u t_{c}(G)$. Also we see that $x^{-1} \phi(x)=f\left(x \gamma_{2}(G)\right) \in Z(G)$. Thus $\phi \in \operatorname{Autcent}(G)$ and hence $\phi \in \operatorname{Aut}_{c}(G) \cap$ Autcent $(G)$. Now $\psi(\phi)=\overline{\alpha_{\phi}}\left(x \gamma_{2}(G)\right)=x^{-1} \phi(x)=x^{-1} a^{-1} x a=$ $f\left(x \gamma_{2}(G)\right)$. Hence

$$
\psi(\phi)=f
$$

Thus $\psi: \operatorname{Aut}_{c}(G) \cap \operatorname{Autcent}(G) \rightarrow \operatorname{Hom}_{c}\left(G / \gamma_{2}(G), Z(G)\right)$ is an isomorphism.

Theorem 10.2. Let $G$ be the group $\phi_{17}\left(1^{6}\right)$ in the isoclinism familiy $\phi_{17}$. Then $\operatorname{Aut}_{c}(G) \neq \operatorname{Inn}(G)$.

Proof. Let $G$ be the group $\phi_{17}\left(1^{6}\right)$. Then

$$
G=<\alpha, \alpha_{1}, \alpha_{2}, \alpha_{3}, \beta, \gamma>
$$

where $\left[\alpha_{i}, \alpha\right]=\alpha_{i+1},\left[\beta, \alpha_{1}\right]=\gamma, \alpha^{p}=\alpha_{1}^{p}=\beta^{p}=\alpha_{i+1}^{p}=\gamma^{p}=1, \quad i=1,2$. Here $\gamma_{2}(G)=<\alpha_{2}, \alpha_{3}, \gamma>$ and $G$ is a nilpotent group of class 3. From $\S 4.1$ of [8], we find that $|Z(G)|=p^{2}$. We observe that $\alpha_{3}, \gamma \in Z(G)$. Since $\alpha_{3}$ and $\gamma$ are the elements of order $p$, therefore $Z(G)=\left\langle\alpha_{3}, \gamma>\right.$. Now $G / Z(G)$ is a nonabelian group of order $p^{4}$ with class 2 and every group of class 2 is flat. Hence 
by lemma 2.7, $\left|Z_{2}(G)\right| /|Z(G)| \geq p^{2}$. But we know that for a non-abelian group $G$ of order $p^{4},|Z(G)| \leq p^{2}$. Therefore $\left|Z_{2}(G)\right| /|Z(G)| \leq p^{2}$. Thus $\left|Z_{2}(G)\right|=$ $p^{4}$. Now $G / \gamma_{2}(G)$ is an elementary abelian p-group of order $p^{3}$. Since every group of order $p^{4}$ enjoys Hasse's principle [10]. Therefore $A_{u t}(G / Z(G))=$ $\operatorname{Inn}(G / Z(G))$. Now by Corollary 2.10, it follows that $\left|A u t_{c}(G)\right|=\mid A u t_{c}(G) \cap$ Autcent $(G)||. G / Z_{2}(G) \mid$.

Let $Z_{1}=[\alpha, G] \cap Z(G), Z_{2}=\left[\alpha_{1}, G\right] \cap Z(G), Z_{3}=[\beta, G] \cap Z(G)$. Then each $Z_{i}$ is a central subgroup of $G$. Now $\alpha_{3}=\left[\alpha_{2}, \alpha\right]$ implies that $\alpha_{3} \in[\alpha, G] \cap$ $Z(G)=Z_{1}$. Similarly $\gamma=\left[\beta, \alpha_{1}\right]$ implies that $\gamma \in\left[\alpha_{1}, G\right] \cap Z(G)=Z_{2}$ and $\gamma \in[\beta, G] \cap Z(G)=Z_{3}$. Hence in each case $\left|Z_{i}\right| \geq p$. Now

$$
\begin{aligned}
\mid \text { Aut }_{c}(G) \cap \operatorname{Autcent}(G) \mid & =\left|\operatorname{Hom}_{c}\left(G / \gamma_{2}(G), Z(G)\right)\right| \\
& =\left|\operatorname{Hom}_{c}\left(\left(<\bar{\alpha}>\oplus<\overline{\alpha_{1}}>\oplus<\bar{\beta}>\right), Z(G)\right)\right| \\
& \geq \text { p.p.p }=p^{3}
\end{aligned}
$$

Hence

$$
\begin{aligned}
\mid \text { Aut }_{c}(G) \mid & =\mid \text { Aut }_{c}(G) \cap \operatorname{Autcent}(G)|\cdot| G / Z_{2}(G) \mid \\
& \geq \frac{p^{3} p^{6}}{p^{4}}=p^{5}
\end{aligned}
$$

Thus $A u t_{c}(G) \neq \operatorname{Inn}(G)$ as $|\operatorname{Inn}(G)|=|G / Z(G)|=p^{4}$.

Let $G$ be the group in the isoclinism families $\phi_{18}-\phi_{21}$. Then in each case $G$ is a nilpotent group of class 3 . We observe that $Z(G)$ and $G / \gamma_{2}(G)$ are elementary abelian p-groups of order $p^{2}$ and $p^{3}$ respectively and $\left|Z_{2}(G)\right|=p^{4}$. Using a similar argument as in the above theorem we find that in each case there are three central subgroups $Z_{i}$ of order $\geq p$. Hence $\mid A u t_{c}(G) \cap$ Autcent $(G) \mid \geq p^{3}$. Since a group of order $p^{4}$ enjoys Hasse's principle, from this we deduce that $\operatorname{Aut}_{c}(G) \neq \operatorname{Inn}(G)$.

If $G$ is a group in the isoclinism family $\phi_{23}$, then $G$ is a nilpotent group of class 4 . We observe that both $Z(G)$ and $G / \gamma_{2}(G)$ are elementary abelian p-groups of order $p^{2}$. Now $G / Z(G)$ is a group of order $p^{4}$ having nilpotency class 3. Now it follows that $\left|Z_{2}(G)\right|=p^{3}$. There are two central subgroups $Z_{i}$ of $G$ such that $\left|Z_{i}\right| \geq p$. This implies that $\mid A u t_{c}(G) \cap$ Autcent $(G) \mid \geq p^{2}$. Using the Corollary 2.10, we find that $\operatorname{Aut}_{c}(G) \neq \operatorname{Inn}(G)$.

If $G$ is a group in the isoclinism family $\phi_{27}$, then $G$ is a nilpotent group of class 4 . We observe that $|Z(G)|=p$ and $G / \gamma_{2}(G)$ is an elementary abelian p-groups of order $p^{3}$. Using the presentation of $G / Z(G)$ from James [8], we find that $\left|Z_{2}(G)\right|=p^{3}$. Again there are three central subgroups $Z_{i}$ each of order 
p. This implies that $\mid A u t_{c}(G) \cap$ Autcent $(G) \mid=p^{3}$. From this we deduce that $\left|A u t_{c}(G)\right|=p^{6}$ and therefore $\operatorname{Aut}_{c}(G) \neq \operatorname{Inn}(G)$.

\section{Nilpotent Groups of Class 2 in which $\gamma_{2}(G)$ is Not Cyclic}

Let $G$ be nilpotent group of class 2 . Then $A u t_{c}(G) \subseteq$ Autcent $(G)$. Hence for a nilpotent group of class $2, \operatorname{Aut}_{c}(G) \cap \operatorname{Autcent}(G)=\operatorname{Aut}_{c}(G)$. In this section we discuss the isoclinism families $\phi_{11}, \phi_{12}, \phi_{13}$ and $\phi_{15}$. We find that a group $G$, lying in any of these isoclinism family is a nilpotent group of class 2 . and from $\S$ of [8], we observe that $\left|x^{G}\right| \leq p^{2}$ for each $x \in G-Z(G)$. We first consider the isoclinism family $\phi_{11}$ and prove the following

Theorem 11.1. Let $G$ be the group in the isoclinism families $\phi_{11}$. Then $\left|A u t_{c}(G)\right|=p^{6}$ and therefore $\operatorname{Aut}_{c}(G) \neq \operatorname{Inn}(G)$.

Proof. Suppose $G$ be the group $\phi_{11}\left(1^{6}\right)$, then $G=<\alpha_{1}, \alpha_{2}, \alpha_{3}, \beta_{1}, \beta_{2}, \beta_{3}>$ where $\left[\alpha_{1}, \alpha_{2}\right]=\beta_{3},\left[\alpha_{2}, \alpha_{3}\right]=\beta_{1},\left[\alpha_{3}, \alpha_{1}\right]=\beta_{2}, \alpha_{i}^{p}=\beta_{i}^{p}=1, \quad i=1,2,3$. Here $\gamma_{2}(G)=<\beta_{1}, \beta_{2}, \beta_{3}>$. Since $\left[\alpha_{i}, \alpha_{j}\right] \neq 1$ and $\left[\alpha_{i}, \beta_{j}\right]=\left[\beta_{i}, \beta_{j}\right]=1$ for $1 \leq$ $i, j \leq 3$, therefore $\beta_{1}, \beta_{2}, \beta_{3} \in Z(G)$. Since $\gamma_{2}(G) \subseteq Z(G)$ and $G$ is non-abelian, $G$ is a nilpotent group of class 2. Hence $A_{u t}(G)=\operatorname{Aut}_{c}(G) \cap \operatorname{Autcent}(G)$. From $\S 4.1$ of [8], we find that $|Z(G)|=p^{3}$. Since $\beta_{1}^{p}=\beta_{2}^{p}=\beta_{3}^{p}=1, Z(G)=<$ $\beta_{1}, \beta_{2}, \beta_{3}>$. Now $G / \gamma_{2}(G)=<\overline{\alpha_{1}}, \overline{\alpha_{2}}, \overline{\alpha_{3}}>$ such that ${\overline{\alpha_{1}}}^{p}={\overline{\alpha_{2}}}^{p}={\overline{\alpha_{3}}}^{p}=1$.

Let $Z_{i}=\left[\alpha_{i}, G\right] \cap Z(G)$. Then each $Z_{i}$ is a central subgroup of $G$. Since $\left[\alpha_{i}, G\right] \subseteq \gamma_{2}(G) \subseteq Z(G)$, therefore $\left|Z_{i}\right|=\left|\left[\alpha_{i}, G\right]\right|=\left|\alpha_{i}^{G}\right|$. Since for each $x \in$ $G-Z(G),\left|x^{G}\right| \leq p^{2}$, therefore $\left|\alpha_{i}^{G}\right| \leq p^{2}$ i.e. $\left|Z_{i}\right| \leq p^{2}$. Now $\beta_{2}=\left[\alpha_{3}, \alpha_{1}\right]$ and $\beta_{3}=\left[\alpha_{1}, \alpha_{2}\right]$, this implies that $\beta_{2}, \beta_{3} \in\left[\alpha_{1}, G\right] \cap Z(G)=Z_{1}$. Hence $\left|Z_{1}\right| \geq p^{2}$. Thus $\left|Z_{1}\right|=p^{2}$ and $Z_{1}=<\beta_{2}, \beta_{3}>$. Similarly $\beta_{1}, \beta_{3} \in\left[\alpha_{2}, G\right] \cap Z(G)=Z_{2}$ and hence $Z_{2}=<\beta_{1}, \beta_{3}>$. Also $\beta_{1}, \beta_{2} \in\left[\alpha_{3}, G\right] \cap Z(G)=Z_{3}$ and hence $Z_{3}=<\beta_{1}, \beta_{2}>$. Now

$$
\begin{aligned}
\left|\operatorname{Aut}_{c}(G)\right| & =\left|\operatorname{Aut}_{c}(G) \cap \operatorname{Autcent}(G)\right| \\
& =\left|\operatorname{Hom}_{c}\left(G / \gamma_{2}(G), Z(G)\right)\right| \\
& =\left|\operatorname{Hom}_{c}\left(<\overline{\alpha_{1}}>\oplus<\overline{\alpha_{2}}>\oplus<\overline{\alpha_{3}}>, Z(G)\right)\right| \\
& =p^{2} \cdot p^{2} \cdot p^{2}=p^{6}
\end{aligned}
$$

Hence $A_{u t}(G) \neq \operatorname{Inn}(G)$ as $\operatorname{Inn}(G)=|G / Z(G)|=p^{3}$.

In the similar fashion, we prove that if $G$ is a group in the isoclinism families $\phi_{13}$ and $\phi_{15}$, then $\left|A u t_{c}(G)\right|$ is $p^{6}$ and $p^{8}$ respectively and hence in both cases $\operatorname{Aut}_{c}(G) \neq \operatorname{Inn}(G)$. 
Theorem 11.2. Let $G$ be the group in the isoclinism families $\phi_{12}$. Then $\operatorname{Aut}_{c}(G)=\operatorname{Inn}(G)$.

Proof. Suppose $G$ be the group $\phi_{12}(2211)_{i}$. Then

$$
G=<\alpha_{1}, \alpha_{2}, \alpha_{3}, \alpha_{4}, \gamma_{1}, \gamma_{2}>,
$$

where $\left[\alpha_{1}, \alpha_{3}\right]=\gamma_{1},\left[\alpha_{2}, \alpha_{4}\right]=\gamma_{2}, \alpha_{1}^{p}=\gamma_{1}$,

$$
\alpha_{2}^{p}=\gamma_{1} \gamma_{2}, \alpha_{3}^{p}=\gamma_{2}, \alpha_{4}^{p}=\gamma_{i}^{p}=1, \quad i=1,2 .
$$

Here $\gamma_{2}(G)=<\gamma_{1}, \gamma_{2}>$. For $1 \leq i \leq 4$ and $1 \leq j \leq 2,\left[\alpha_{i}, \gamma_{j}\right]=\left[\gamma_{1}, \gamma_{2}\right]=1$. This implies that $\gamma_{1}, \gamma_{2} \in Z(G)$. Thus $\gamma_{2}(G) \subseteq Z(G)$ and hence $G$ is a nilpotent group of class 2. Now $A_{u t}(G)=\operatorname{Aut}_{c}(G) \cap \operatorname{Autcent}(G)$. From $\S 4.1$ of [8], we find that $|Z(G)|=p^{2}$. Since $\gamma_{1}^{p}=\gamma_{2}^{p}=1$, therefore $Z(G)=<\gamma_{1}, \gamma_{2}>$. Let $Z_{i}=\left[\alpha_{i}, G\right] \cap Z(G)$. Then each $Z_{i}$ is a central subgroup of $G$. Since $\left[\alpha_{i}, G\right] \subseteq \gamma_{2}(G) \subseteq Z(G)$, therefore $\left|Z_{i}\right|=\left|\left[\alpha_{i}, G\right]\right|=\left|\alpha_{i}^{G}\right|$. Since for each $x \in G-Z(G),\left|x^{G}\right| \leq p^{2}$, therefore $\left|\alpha_{i}^{G}\right| \leq p^{2}$ i.e. $\left|Z_{i}\right| \leq p^{2}$. Since $\alpha_{1}$ commutes with $\alpha_{2}, \alpha_{4}, \gamma_{1}, \gamma_{2}$ and $\left[\alpha_{1}, \alpha_{3}\right]=\gamma_{1}$, therefore $\left[\alpha_{1}, G\right]=<\gamma_{1}>$. But then $Z_{1}=<\gamma_{1}>$. Similarly $\alpha_{2}$ commutes with $\alpha_{1}, \alpha_{3}, \gamma_{1}, \gamma_{2}$ and $\left[\alpha_{2}, \alpha_{4}\right]=$ $\gamma_{2}$, therefore $\left[\alpha_{2}, G\right]=<\gamma_{2}>$. But then $Z_{2}=<\gamma_{2}>$. In the same fashion we find that $Z_{3}=\left[\alpha_{3}, G\right] \cap Z(G)=<\gamma_{1}>$ and $Z_{4}=\left[\alpha_{4}, G\right] \cap Z(G)=<\gamma_{2}>$. Thus $\left|Z_{i}\right|=p$ for each i, $1 \leq i \leq 4$. Since $\gamma_{2}(G) \subseteq Z(G)$ and $\left|\gamma_{2}(G)\right|=|Z(G)|=p^{2}$, therefore $\gamma_{2}(G)=Z(G)$. Here $G / \gamma_{2}(G)=<\overline{\alpha_{1}}, \overline{\alpha_{2}}, \overline{\alpha_{3}}, \overline{\alpha_{4}}>$ such that ${\overline{\alpha_{1}}}^{p}=$ ${\overline{\alpha_{2}}}^{p}={\overline{\alpha_{3}}}^{p}={\overline{\alpha_{4}}}^{p}=1$. Now

$$
\begin{aligned}
\left|\operatorname{Aut}_{c}(G)\right| & =\left|\operatorname{Aut}_{c}(G) \cap \operatorname{Autcent}(G)\right| \\
& =\left|\operatorname{Hom}_{c}\left(G / \gamma_{2}(G), Z(G)\right)\right| \\
& =\prod_{i=1}^{4}\left|\operatorname{Hom}_{c}\left(<\overline{\alpha_{i}}>, Z(G)\right)\right| \\
& =p \cdot p \cdot p \cdot p=p^{4}
\end{aligned}
$$

Thus $\operatorname{Aut}_{c}(G)=\operatorname{Inn}(G)$ as $|\operatorname{Inn}(G)|=|G / Z(G)|=p^{4}$.

Remark: Out of 43 isoclinism families, unfortunately we are unable to find relation between $A u t_{c}(G)$ and $\operatorname{Inn}(G)$ for a group $G$ lying in the isoclinism families $\phi_{30}, \phi_{37}$ and $\phi_{39}$.

\section{References}

[1] J.E. Adney, T.Yen, Automorphisms of a p-group, Illinois J. Math, 9 (1965),137-143. 
[2] A.R. Camina, Some conditions which almost characterize Frobenious groups, Israel journal of Mathematics, 31 (1978), 153-160.

[3] D.Rex, C. M. Scoppola, On Camina groups of prime power order, Journal of algebra, 181(1996),787-802.

[4] M. Fuma,Y. Ninomiya, Hasse's principle for finite p-groups with cyclic subgroups of index $p^{2}$, Math J.Okayama University, 46 (2004), 31-38.

[5] D.Gumber, Mahak Sharma, On central automorphisms of finite p-groups, Archiv der mathematike, 9(2011), 152-182.

[6] P. Hall, The classification of prime power groups, J.fur die reine und angewandte Mathematik,182 (1940), 130141.

[7] M. Hertweck, Contributions to the integral representation theory of groups, Habilitationsschrift (University of Stuttgart),(2004)

[8] R. James, The groups of order $p^{6}$ ( $p$ an odd prime), Math. comp., 34 (1980), 613-637.

[9] M. Kumar, L. R. Vermani, Hasse's principle for extra-special p-groups, Proc. Japan Acad., 76 A (2000), 123-125.

[10] M. Kumar, L. R. Vermani, Hasse's principle for groups of order $p^{4}$, Proc. Japan Acad., 77 A(2001), 95-98.

[11] M. Kumar, L. R. Vermani,On automorphisms of some p-groups, Proc. Japan Acad., 78 A (2002), 46-50.

[12] T. Ono, On Shafarevich Tate set for profinite groups, Proc. Japan Acad., 75 A (1999),97-98.

[13] T. Ono, H. Wada,Hasse's Principle for symmetric and alternating groups, Proc.Japan Acad, 75 A (1999), 61-62.

[14] H. Tandra, W. Moran,Flatness conditions on finite p-groups, (Comm. Algebra), 32(2004), 2215-2224.

[15] M. K. Yadav, On automorphisms of some p-groups, Proc. Indian Acad. Sci., 118(2008), 1-11. 
